\title{
Investigating the Role of the Electrostatic Force in Spore Adhesion by Microscopy
}

\author{
I. Lee, ${ }^{* * *}$ E. Chung, ${ }^{* * *}$ H. Kweon, ${ }^{* * *}$ S. Yiacoumi, ${ }^{* * *}$ and C. Tsouris****** \\ * Department of Electrical Engineering \& Computer Science, University of Tennessee, Knoxville, \\ TN 37996 \\ ** Oak Ridge National Laboratory, Oak Ridge, Tennessee 37831 \\ *** School of Civil and Environmental Engineering, Georgia Institute of Technology, Atlanta, GA \\ 30332
}

Understanding the interaction and adhesion of bacterial spores at surfaces-the first step for the subsequent germination - is very important in the fields of bioprocess engineering, medicine, and biological decontamination. Bacterial spore adhesion to inert surfaces is generally believed to involve surface hydrophobicity [1,2], surface polarity [3], surface electrical charge [4,5], and van der Waals (vdW) interactions.

Previously, we investigated the adhesion force between a Bacillus thuringiensis $(B t)$ spore and a gold planar surface using atomic force microscopy (AFM) measurements and calculations based on mechanistic modeling [6]. Although spores are known to attain electrostatic charge on their surface [7], previous experiments were conducted using a $B t$ spore and a gold substrate at conditions for which the electrostatic force could be ignored as a component of the adhesion force. Theoretical and experimental results were comparable for a $B t$ spore and the gold surface system. The measurements between the $B t$ spore and a mica surface showed a significant deviation from calculated results, which is believed to be due to the electrostatic force. This indicated that electrostatic force may play an important part in particle adhesion. In a recent study, we found out that the electrostatic force, including Coulombic and image forces, can account for up to $40 \%$ of the total adhesion force between a bacterial spore and a planar surface [8].

We have measured the electrostatic potential of $B t$ spores on various substrates under various relative humidity (RH) values (examples are shown in FIG.1 and TABLE 1) by using our combined atomic force microscopy (AFM) and scanning surface potential microscopy (SSPM) technique. SSPM, also known as Kelvin probe force microscopy (KPFM), has been previously used to map the surface morphology and the corresponding local electrostatic potential in nanometer resolution. The total adhesion force was measured through AFM force curves. The results were used to compare with those from a theoretical model. The electrostatic force for four different relative humidity levels on mica is shown in FIG. 2. According to the calculations, the repulsive electrostatic force canceled a part of the attractive capillary and vdW forces. Approximately $60 \%$ of the sum of the two attractive forces at $20 \%$ relative humidity, and $25 \%$ of the attractive force at $80 \%$ relative humidity, were canceled by the electrostatic force. FIG. 3 depicts the measured adhesion force between the $B t$ spore and gold surface by AFM and the calculated adhesion force consisting of three components: capillary, van der Waals, and electrostatic image forces. The calculated image force is approximately $10 \%$ of the total adhesion force for the relative humidity range $20 \%$ to $80 \%$. The added component of the image force improved the theoretical predictions, however, the discrepancy between the experimental values and model calculations for the gold surface system is greater compared to that for the mica surface system. This difference may due to the surface roughness of the gold surface. 
References

[1] U. Husmark and U. Ronner, Biofouling 5 (1992) 335.

[2] U. Hüsmark and U. Ronner, Biofouling 7 (1993) 57.

[3] L. Bouiange-Petermann et al., J. Adhes. Sci. Technol. 7 (1993) 221.

[4] U. Husmark and U. Ronner, J. Appl. Bacteriol. 69 (1990) 557.

[5] S. H. Flint et al., J. Food Eng. 43 (2000) 235.

[6] E. Chung et al., Environ. Sci. Technol. 44 (2010) 290.

[7] U. Husmark et al., J. Appl. Bacteriol. 69 (1990) 557.

[8] E. Chung et al., S. Environ. Sci. Technol. 44 (2010) 6209.

TABLE 1. SSPM measurements of Bt spore on gold substrate under different relative humidity (RH) levels.

\begin{tabular}{|c|c|}
\hline RH (\%) & $B t$ spore potential $(\mathrm{V})$ \\
\hline 0 & -0.454 \\
\hline 10 & -0.445 \\
\hline 20 & -0.439 \\
\hline 30 & -0.423 \\
\hline 40 & -0.409 \\
\hline 50 & -0.392 \\
\hline 60 & -0.373 \\
\hline 70 & -0.362 \\
\hline 80 & -0.354 \\
\hline
\end{tabular}

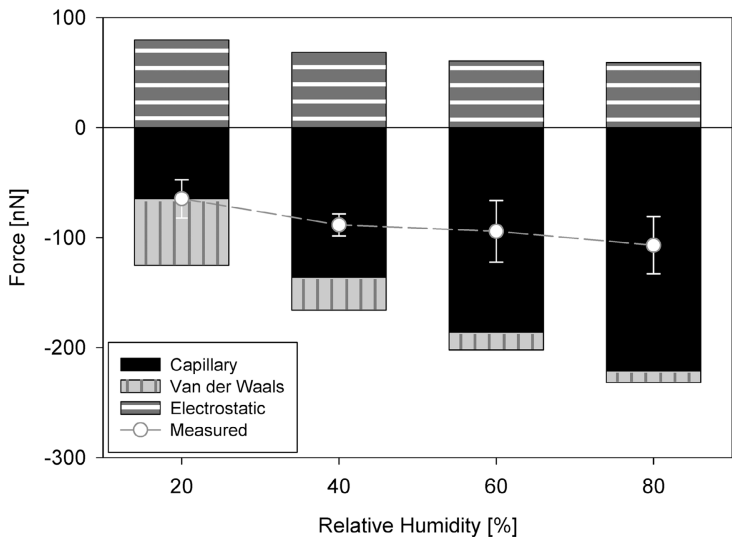

FIG. 2. Measured adhesion force by AFM between a $B t$ spore and a mica surface and model calculation of its components: capillary, van der Waals, and electrostatic forces [8].
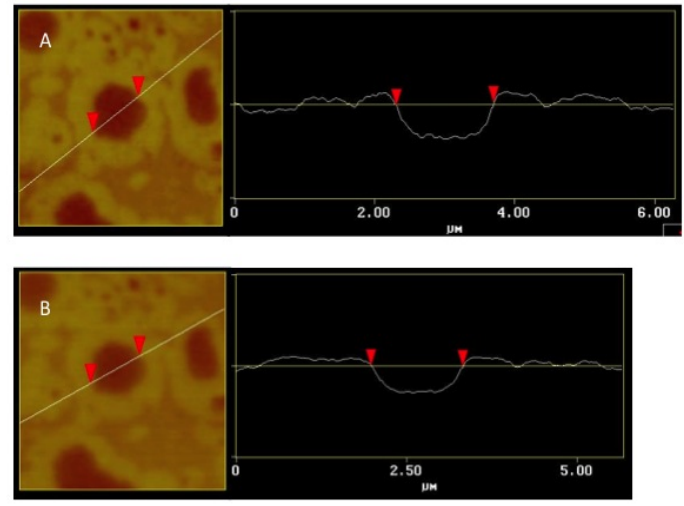

FIG. 1. SSPM cross section analysis of one of the spores on gold substrate at (A) $0 \%$ and (B) $80 \% \mathrm{RH}$.

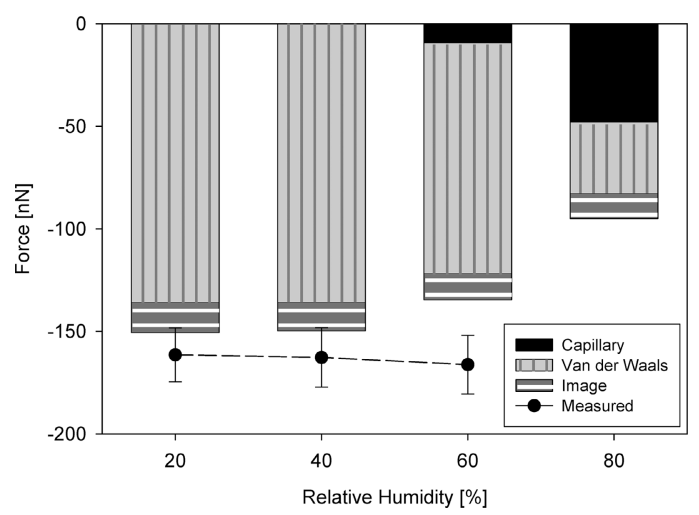

FIG. 3. Measured adhesion force by AFM between a $B t$ spore and a gold surface and model calculation of its components: capillary, van der Waals, and electrostatic image forces [8]. 